Hautarzt 2021 · 72:1071-1078

https://doi.org/10.1007/s00105-021-04868-4

Angenommen: 6. Juli 2021

Online publiziert: 30 . Juli 2021

(c) Springer Medizin Verlag GmbH, ein Teil von Springer Nature 2021

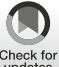

\section{Therapie der atopischen Dermatitis mit Dupilumab}

\author{
Eine retrospektive Kohortenanalyse aus der \\ dermatologischen Praxis
}

Sigbert Jahn · Julia Föhr · Evangelia Diamanti · Matthias Herbst

Dermatologische Praxis Dr. Herbst \& Kollegen, Darmstadt, Deutschland

\title{
Zusammenfassung
}

Wir präsentieren die Ergebnisse einer retrospektiven Datenauswertung von 44 Patienten einer Praxiskohorte, deren atopische Dermatitis (AD) über bis zu 3 Jahre mit dem IL(Interleukin)-4/13-Rezeptor-Antikörper Dupilumab behandelt wurde. Die Betreuung erfolgte in den letzten 21 Monaten in einer Spezialsprechstunde Immundermatologie, in der die aufwendige Dokumentation realisiert werden konnte. Die Charakteristika der Kohorte hinsichtlich Alters- und Geschlechterverteilung, Schwere und Dauer der Erkrankung sind vergleichbar mit den großen Patientenkollektiven der Zulassungsstudien. Die therapeutische Effizienz unter Praxis-Alltagsbedingungen erwies sich als sehr gut (Anteil Patienten EASI [Eczema Area and Severity Index] 50, 75, 90 nach 16 Wochen: 94, 84, 60\%) und anhaltend (86\% EASI 90 nach 52 Wochen Therapie). Etwa die Hälfte unserer Patienten hatte anamnestisch oder bei Therapiebeginn eine Beteiligung der Gesichtshaut und/oder Augen. Diese Patientengruppe erwies sich als betreuungsintensiver, weil die Gesichts- und periorbitale Dermatitis sowie begleitende Konjunktividen verzögert abheilten, zu Rezidiven neigten und häufig einer zusätzlichen topischen Therapie bedurften. Wir haben keine schweren Nebenwirkungen in den hier ausgewerteten 48 Patientenjahren festgestellt. Dupilumab erwies sich als sichere und effiziente Therapie für die atopische Dermatitis in der dermatologischen Praxis.

\section{Schlüsselwörter}

Gesichtsdermatitis · Immundermatologie · Interleukin-4/13-Blockade · Immunmodulation · Periorbitale Dermatitis

Patienten mit atopischer Dermatitis (AD) stellen sich häufig in der dermatologischen Sprechstunde vor [16]. Hinsichtlich des sehr komplexen und individuell äußerst heterogenen Krankheitsverlaufes, basierend auf unterschiedlichen Pathogenesemechanismen [14], darf man schlussfolgern, dass es "die" AD vielleicht gar nicht gibt. Eine gestörte Funktion der Hautbarriere verbunden mit der Aufnahme von Allergenmaterial und ein entsprechend sensibilisiertes Immunsystem [14] befördern sich gegenseitig. Dies wird von psychosomatisch-psychodermatologischen Faktoren [12] begleitet. Mit zunehmendem Verständnis der vielfältigen pathogenetischen Faktoren und Mechanismen wächst die Zahl der Interventionsmöglichkeiten, was zu einer Viel- zahl von therapeutischen Entwicklungen führte und führt $[5,17]$. Dazu gehören der bereits seit 3,5 Jahren verfügbare monoklonale Anti-IL(Interleukin)4-/IL13Rezeptor-Antikörper Dupilumab und die JAK(Januskinase)-Inhibitoren $[5,28] \mathrm{mit}$ dem seit Dezember 2020 verfügbaren Baricitinib. Es fällt nicht schwer vorauszusehen, dass in wenigen Jahren ein ganzes therapeutisches Arsenal für die Behandlung der $A D$ bereitstehen wird und, ähnlich wie bei der Psoriasis, die Frage zu stellen sein wird, welches Präparat für welchen Patienten am besten geeignet ist. Klinische Beobachtungen, die im Therapieverlauf erhoben und später (retrospektiv) ausgewertet werden, also „Real World Data“ in Form einer retrospektiven Kohortenstudie [9, 13], werden hier 
Tab. 1 Charakterisierung einer Patientenkohorte mit atopischer Dermatitis (AD), die in der Praxis mit Dupilumab behandelt wurde

\begin{tabular}{|l|l|}
\hline Gesamtzahl Patienten & 44 \\
\hline $\begin{array}{l}\text { Geschlechterverteilung weib- } \\
\text { lich:männlich }\end{array}$ & $25: 19$ \\
\hline Alter: Median; Durchschnitt (Jahre) & $36 ; 38$ \\
\hline $\begin{array}{l}\text { Patienten unter 18 Jahren bei } \\
\text { Therapiestart }\end{array}$ & $4 / 44$ \\
\hline $\begin{array}{l}\text { Dauer der Erkrankung: Median; } \\
\text { Durchschnitt (Jahre) }\end{array}$ & $25 ; 28$ \\
\hline $\begin{array}{l}\text { Late-onset-AD (Erstmanifestation } \\
\text { im Erwachsenenalter) }\end{array}$ & $8 / 44$ \\
\hline $\begin{array}{l}\text { EASI bei Therapiestart; Durch- } \\
\text { schnitt }\end{array}$ & $\begin{array}{l}8-57 ; \\
21\end{array}$ \\
\hline $\begin{array}{l}\text { Schwerster EASl in der Anamnese; } \\
\text { Durchschnitt }\end{array}$ & $8-57 ;$ \\
25
\end{tabular}

Atopische/allergische Begleiterkrankungen

\begin{tabular}{|l|l}
\hline - Rhinokonjunktivitis & 17
\end{tabular}

- Allergisches Asthma bronchiale 14

\begin{tabular}{l|l}
\hline - Polyposis nasi & 1 \\
\hline
\end{tabular}

\begin{tabular}{|l|l}
\hline - Nahrungsmittelallergien & 3 \\
\hline
\end{tabular}

\begin{tabular}{|l|l|}
\hline - Urtikaria & 4 \\
\hline Lebensqualität (DLQI, Höchst- & $5-29$
\end{tabular}

wert 30)

\begin{tabular}{l|l}
\hline - Median; Mittelwert & 21;20 \\
\hline
\end{tabular}

\begin{tabular}{ll|}
\hline Patienten mit starker Gesichtsbe- & $19 / 44$
\end{tabular} teiligung mit/ohne Konjunktivitis (anamnestisch oder/und bei Therapiestart)

\begin{tabular}{|l|l|}
\hline Behandlungsdauer (Monate) & $1-39$ \\
\hline $\begin{array}{l}\text { Patientenjahre der Dupilumab-Be- } \\
\text { handlung in dieser Kohorte }\end{array}$ & 48 \\
\hline
\end{tabular}

\begin{tabular}{l|l|}
\hline \multicolumn{2}{|l|}{ Vorbehandlungen } \\
\hline - Cyclosporin & $3 / 44$ \\
\hline $\begin{array}{l}\text { - Systemische Kortikosteroide } \\
\text { kurzzeitig im Intervall }\end{array}$ & $12 / 44$ \\
\hline $\begin{array}{l}\text { - Mehrmonatig systemisch Pred- } \\
\text { nisolon in mittlerer Dosis }\end{array}$ & $2 / 44$ \\
\hline $\begin{array}{l}\text { EASI Eczema Area and Severity Index, DLQI } \\
\text { Dermatology Life Quality Index }\end{array}$
\end{tabular}

gezeigt. Möglicherweise können solche Daten helfen, Neuentwicklungen schnell im Verordnungsalltag einzuordnen und entsprechende Therapiealgorithmen zu etablieren. Dieser Aufgabe sollten sich in der kommenden Zeit Dermatologen in Klinik und Praxis stellen [28].
Tab. 2 Ig(Immunglobulin)E und Allergenspezifitäten bei Patienten mit atopischer Dermatitis (AD), die mit Dupilumab therapiert wurden

\begin{tabular}{|l|l|}
\hline $\begin{array}{l}\text { IgE erhöht (Zahl } \\
\text { Patienten) }\end{array}$ & $\begin{array}{l}\text { IgE nicht erhöht } \\
\text { (Zahl Patienten) }\end{array}$ \\
\hline 36 & 5 \\
\hline Allergenspezifitäten & Allergenspezifitäten \\
\hline 33 positiv & 4 positiv \\
\hline 3 negativ & 1 negativ \\
\hline
\end{tabular}

\section{Resultate}

\section{Dupilumab-Patientenkohorte}

In unserer Praxis werden pro Jahr etwa 220 Patienten mit AD (inklusive atopischem Handekzem) völlig unterschiedlicher Schweregrade und mit sehr unterschiedlichen Krankheitsgeschichten vorstellig. Im Rahmen einer Spezialsprechstunde Immundermatologie wurden innerhalb von 21 Monaten 37/44 der hier charakterisierten Patienten auf die Therapie mit Dupilumab eingestellt. Bei 4 Patienten war die Therapie in unserer Praxis vorher gestartet worden. Wir übernahmen die Therapie bei 3 Patienten, die in Kliniken auf Dupilumab eingestellt worden waren. Bei einer Patientin kam es in einer Phase-III-Studie mit einem JAKInhibitor zu einem schweren Rezidiv, das wir nach Beendigung der Studienmedikation mit Dupilumab weiter behandelten. Drei Patienten wurden durch andere dermatologische Praxen mit der Bitte um Therapieeinstellung überwiesen. Viermal stellten sich Patienten auf Anraten Dupilumab-behandelter Patienten vor. Mehrere Patienten wurden vom Hausarzt explizit mit dem Hinweis auf Dupilumab an uns überwiesen. Bei 15 Patienten gab es eine aktuell andauernde oder vorherige systemische Vorbehandlung mit Cyclosporin (3) oder systemischen Kortikosteroiden im Intervall (12), 2 Patienten waren mehrmonatig auf einer mitteldosigen Prednisolon-Therapie (5 und $10 \mathrm{mg} / \mathrm{Tag})$. Vierzehn Patienten waren in ihrer Krankheitsgeschichte lange Zeit ohne dermatologische Behandlung und erhielten weder topische, UV-Licht- oder systemische Therapien. Die Basisdaten unserer Patientenkohorte sind in $\mathbf{0}$ Tab. 1 dokumentiert (Dokumentationsschluss 31.05.2021).
Alle Patienten mit $A D$, bei denen eine Therapie mit Dupilumab gestartet wurde, werden aktuell in der Spezialsprechstunde Immundermatologie betreut. Vor Therapiestart (Woche 0) erfolgten die Bestimmung des EASI (Eczema Area and Severity Index), die Abfrage des Juckreiz-NRS(numerische Rating-Skala 0-10)Scores sowie die Erhebung der Lebensqualität (DLQI[Dermatology Life Quality Index]-Fragebogen). Ebenso wurde für jeden Patienten ein anamnestischer EASI bestimmt, indem Daten aus früheren Konsultationen in unserer oder anderen Praxen, Angaben aus Klinikbriefen, Handyfotos und Berichte der Patienten herangezogen wurden. Der DurchschnittsEASI bei Therapiestart lag unwesentlich unter dem anamnestisch erhobenen Wert (21 vs. 25). Bei 16 der 37 von uns selbst eingestellten Patienten war der Schweregrad bei Therapiestart der bisher höchste im Krankheitsverlauf. Die Schwere der Erkrankung wurde durch Fotoaufnahmen typischer Effloreszenzen und deren Ausbreitung dokumentiert. Es erfolgte die Erhebung des Impfstatus. Bei allen Patienten wurde eine einmalige Analyse von Basisblutwerten (Blutbild, Leber- und Nierenfunktionsparameter) sowie ein REAST (Reversed Enzyme Allergo Sorbent Test) (s. unten) vorgenommen.

Bei 8 Patienten trat die AD erst im Erwachsenenalter auf (4-mal im 20. bis 30., 2-mal im 30. bis 50., 2-mal im 60. bis 70. Lebensjahr). Drei Patienten berichteten, in der Kindheit erkrankt gewesen zu sein, gefolgt von einer jahrelangen Remission. Sie erlitten Rezidive jenseits des 40. Lebensjahres. Es wurde eine Patientin mit niedrigem EASI (Beugenekzem), jedoch massiver Gesichtsbeteiligung mit häufiger Konjunktivitis und oralem Nahrungsmittelallergiesyndrom nach Absprache mit der Krankenkasse behandelt.

Bei einem Teil der Patienten bestanden atopisch allergische Begleiterkrankungen (- Tab. 1), die wir anamnestisch oder dank der Angaben in Arzt- und Klinikbriefen (17/44 Rhinokonjunktivitis) oder anhand von Handyfotos diagnostizierten (4/44 Urtikaria). Die 3 Nahrungsmittelallergien (ein orales Allergiesyndrom) wurden nicht durch uns, sondern in Kliniken diagnostiziert. Die Diagnose allergisches Asthma bronchiale wurde immer durch 
Tab. 3 Ansprechraten (Erreichen der jeweiligen EASI [Eczema Area and Severity Index]-Reduktion) im Zeitverlauf

\begin{tabular}{|c|c|c|c|c|c|c|}
\hline Woche & EASI 50 & EASI 75 & EASI 90 & DLQI $50^{\mathrm{a}}$ & DLQI 75a & Pruritusreduktion $^{b}$ \\
\hline 4 & $34 / 39$ & $27 / 39$ & $13 / 39$ & $20 / 28$ & $12 / 28$ & $27 / 37$ \\
\hline 8 & $34 / 36$ & $30 / 36$ & $16 / 36$ & $22 / 28$ & $18 / 28$ & $31 / 34$ \\
\hline 16 & $30 / 32$ & $27 / 32$ & $19 / 32$ & $21 / 26$ & $18 / 26$ & $29 / 30$ \\
\hline 52 & $22 / 22$ & $19 / 22$ & $13 / 22$ & $16 / 19$ & $16 / 19$ & $19 / 21$ \\
\hline \multicolumn{7}{|c|}{$\begin{array}{l}\text { ¿Verbesserung der Lebensqualität im Therapieverlauf, dokumentiert anhand der 50- oder } 75 \text { \%igen } \\
\text { Reduktion des DLQI(Dermatology Life Quality Index)-Wertes gegenüber Ausgangswert } \\
\text { bJuckreizreduktion anhand Reduktion des NRS(numerische Rating-Skala)-Ausgangswertes um } 4 \text { Punk- } \\
\text { te (2 Patienten mit <4 wurden nicht ausgewertet) }\end{array}$} \\
\hline
\end{tabular}

den (mit) betreuenden Pulmologen gestellt. Alle 14 Patienten hatten eine entsprechende Komedikation. Die Diagnose Polyposis nasi wurde HNO(Hals-Nasen-Ohren)-ärztlich gestellt.

\section{Laborwerte: IgE-Spiegel}

Bei nahezu allen Patienten mit Verdacht auf $A D$ erfolgte die Bestimmung des Gesamt-Ig(Immunglobulin)E im Serum (ELISA [Enzyme-linked Immunosorbent Assay]) sowie der Antigenspezifitäten (REAST). Vergleiche unterschiedlicher Laboranalysen sind schwierig. In unseren Partnerlabors wird ein Wert $>100 \mathrm{IU} / \mathrm{ml}$ als erhöhtes $\lg \mathrm{E}$ angegeben. In einem Labor werden Werte $>1000 \mathrm{IU} / \mathrm{ml}$ nicht weiter titriert. Wir charakterisieren die Patienten unserer Kohorte als „IgE erhöht" bei Werten $>200 \mathrm{IU} / \mathrm{ml}$ und als „Allergenpositiv“ bei >Klasse 2 (ab "mäßig positiv“) für 2 unterschiedliche Allergengruppen. In der Regel werden 8 Spezifitäten getestet. Dazu gehören immer die Baumpollen (Birke, Hasel, Erle), Mix von frühblühenden Gräsern, Roggen, Hausstaubmilben (Dermatophagoides pteronyssinus et farinae), Schimmelpilzmischung, Tierhaare oder -epithelien. Die IgE-Spiegel im Serum unserer Patienten variierten von $<30 \mathrm{lU} / \mathrm{ml}$ bis $>15.000 \mathrm{IU} / \mathrm{ml}$. Soweit das überhaupt beurteilbar ist, haben wir keine Korrelationen zwischen IgE-Spiegeln und Schweregrad (EASI) festgestellt. In etwa der Hälfte der Patienten gab es eine nahezu identische Allergenspezifität im Pricktest (nicht gezeigt). Bei 4/5 Patienten mit $\lg \mathrm{E}<100 \mathrm{IU} / \mathrm{ml}$ wurden jeweils deutlich positive Spezifitäten auf Birkenpollen gemessen. Die Ergebnisse wurden in - Tab. 2 zusammengefasst.

\section{Therapieverlauf}

Die Erhebung von EASI, DLQI, PruritusScore sowie die Fotodokumentation erfolgten in Woche 0 (Therapiestart), 4, 8 und 16. In Woche 16 kam es zum „Therapieentscheid", der gemeinsam von Patienten, MFAs (medizinische Fachangestellte) und Ärzten besprochen wurde. Im Falle der Therapiefortsetzung erfolgte die Dokumentation des Verlaufes (s. oben) 1-mal pro Quartal. Die - Tab. 3 zeigt die Daten zur Wirksamkeit von Dupilumab im Zeitverlauf, dokumentiert anhand desEASI, der Pruritusreduktion (> minus 4 Punkte gegenüber Ausgangswert) sowie der Verbesserung der Lebensqualität (DLQI-Reduktion um 50 bzw. $75 \%$ ). Für etwa die Hälfte der Patienten konnte eine Analyse $1 \mathrm{Jahr}$ nach Therapiestart dokumentiert werden. Die Mehrzahl der Patienten reagierte mit sehr schnellem Ansprechen. Etwa $90 \%$ der Patienten erreichten die Halbierung des EASI nach 4 Wochen. Bei 21/34 Patienten wurde ein EASI 50 bereits nach 2 Wochen festgestellt. Parallel dazu kam es zu einer raschen Reduktion des Pruritus und zu einer Verbesserung der Lebensqualität (- Tab. 3).

\section{Arzneimittelsicherheit}

Bei etwas weniger als der Hälfte unserer Patienten (19/44) ergab sich in der Anamneseerhebung, aus Berichten über Klinikaufenthalte, der klinischen Untersuchung bei Therapiestart und/oder anhand von Fotos aus Patientenhandys ein starker Ekzembefall der Gesichtshaut, häufig besonders imponierend als periorbitale Dermatitis, begleitet in 8/19 Fällen von einer Konjunktivitis. Das Geschlechterverhältnis der Patientengruppe mit Gesichtsbeteiligung war 12 Frauen und 7 Männer. Es wurde keine Beziehung zu IgE-Höhe und -Spezifität festgestellt. Insgesamt 8/19 Patienten gaben eine Saisonalität der Verschlechterung der Gesichtshaut im zeitigen Frühjahr an. Für alle 19 Patienten mit einer anamnestisch oder bei Therapieeinstellung bestehenden Beteiligung der Gesichtshaut galt: Die Abheilung des Ekzems im Gesicht erfolgte teilweise stark verzögert. Es bestanden also hier noch Anzeichen der akuten Dermatitis, wenn der übrige Körper bereits zu EASI 90 tendierte. Wir haben keinen Fall eines Auftretens einer Gesicht- und Augenbeteiligung gesehen, wo dies im bisherigen Krankheitsverlauf nicht schon bekannt gewesen wäre. Für 6 der 19 Patienten organisierten wir eine augenärztliche Mitbetreuung. Hier wurde jeweils eine Konjunktivitis diagnostiziert und mit steroidhaltigen Tropfen therapiert. Bei allen Patienten heilten auch die Hauterscheinungen im Gesicht unter Dupilumab ab. Jedoch war der Zeitraum, in dem dies erreicht wurde (18 bis 28 Wochen) länger, und die Patienten neigten zu Rezidiven. Von den 44 Patienten der Kohorte berichteten 6 (alle weiblich, alle jünger als der Altersmedian der Gruppe) von der Beobachtung einer "nachlassenden Wirkung" des Medikamentes, gekennzeichnet durch Wiederauftreten einer schwachen Symptomatik (Juckreiz, Erythem an typischen Prädilektionsstellen) etwa 3 Tage vor der nächsten (11 Tage nach der letzten) Injektion. Es wurden keine Lokalreaktionen nach Injektion beschrieben - weder mit der Fertigspritze noch mit dem PEN-Injektor. In den ersten 3 Monaten der Therapie erfolgte die 2-wöchentliche Injektion nahezu ausnahmslos in der Praxis und durch dieselbe MFA. Ab Quartal 2 bietet diese MFA den Patienten eine Spritzenschulung an. Nachfolgend verabreichten sich $80 \%$ der Patienten das Präparat selbst oder wurden von einem Familienmitglied in häuslicher Umgebung gespritzt. Dabei präferieren $50 \%$ den PEN, die andere Hälfte die Fertigspritze. Wir haben keine systemischen Nebenwirkungen (Infektion, Allergie) beobachtet.

\section{Besondere Kasuistiken}

Bei 4 Patientinnen lag eine anamnestisch bekannte, klinisch relevante und labor- 
medizinisch nachgewiesene spezifische Sensibilisierung vor: Hundeepithel, Pferdehaare, Nahrungsmittelallergene (auswärts charakterisiert). Alle 4 Patientinnen zeigten einen guten Heilungsverlauf des Ekzems unter Dupilumab und nahmen selbstständig „Expositionen“ vor, die zuvor über viele Jahre vermieden worden waren: Hund streicheln, Reiten, Möhreneintopf bzw. Erdbeere zu sich nehmen. In keinem der Fälle wurden eine Verschlechterung des Hautzustandes oder andere allergische Reaktionen beschrieben.

Der am meisten protrahierte Therapieverlauf wurde bei einem 63-jährigen Patienten beobachtet. Dieser hatte bei Erstvorstellung in der Praxis eine erst seit 1 Jahr bestehende AD mit EASI über 40, $\operatorname{lgE}>1000 \mathrm{IU} / \mathrm{ml}$ und leichter Eosinophilie. Die Hauterkrankung war im zeitlichen Zusammenhang mit einem schweren Psychotrauma (Zeuge eines Personenunfalls) erstmals aufgetreten. Als klinische Besonderheit sahen wir eine massive Impetiginisierung der teilweise pruriginösen Ekzemstellen und folglich stark erhöhte Entzündungsparameter im Blut. Die antibiotische Therapie ergab zunächst keinen Effekt (Keime erwiesen sich als resistent gegenüber Doxycyclin). Erst nach Umstellung auf Clindamycin kam es zur Besserung. Bei diesem Patienten erreichten wir erst zu Woche 16 (Zeitpunkt des Therapieentscheids) eine EASI 50-Besserung und ein Abklingen des vorher als äußerst quälend empfundenen Pruritus.

Eine 54-jährige Patienten mit lebenslanger Krankheitsgeschichte und mehrfachen stationären Aufenthalten war wiederholt mit systemischen Kortikosteroiden, 4 Monate mit Xolair (Omalizumab Novartis Nürnberg, Deutschland) ("off label“ in der Klinik) und knapp 5 Jahre lang mit Cyclosporin (per os: 2,5 mg/kgKG [Körpergewicht]) behandelt worden und zunächst nicht bereit, an der laufenden Therapie etwas zu ändern, weil der Hautzustand „gut sei“ (EASI um 10, bei früheren Schüben über 45). Erst Auffälligkeiten in den Blutwerten, eine Hypertonie sowie die sich zuspitzende Coronavirus-Pandemie bewogen die Patientin, unserem Rat zu einer Dupilumab-Therapie zu folgen. Auf eigenen Wunsch nahm die diesbezüglich erfahrene Patientin im Urlaub eine Dosisreduktion bis $1 \mathrm{mg} / \mathrm{kgKG}$ vor, gefolgt von einer 4-wö- chigen Phase ohne Therapie, bevor wir Dupilumab gemäß Fachinformation mit 2-mal 300 mg s.c. starteten. Eine Woche später kam es zu einem schweren Rezidiv der atopischen Dermatitis (EASI um 20, Juckreiz VAS [visuelle Analogskala] 9/10), das aber nach der 2. Injektion, also etwa in Woche 3 der Dupilumab-Therapie, begleitet von topischer Steroidanwendung, abklang. Nach nunmehr 5 Monaten auf Dupilumab besteht nahezu Erscheinungsfreiheit (EASI <2).

Drei Patientinnen kommunizierten etwa 5 bis 6 Monate nach Therapiestart (bei sehr gutem Hautzustand) ihren Kinderwunsch. Gemäß Fachinformation informierten wir über die Möglichkeit der Weitertherapie oder einer Unterbrechung der Dupilumab-Therapie und sofortiger Wiederaufnahme bei entsprechendem Hautzustand. Erfreulicherweise waren zum Zeitpunkt der Anfertigung des Manuskriptes 2 Patientinnen schwanger. Beide entschlossen sich zur Therapieunterbrechung. Wir betreuen engmaschig, um bei einem Rezidiv schnell reagieren zu können, und stehen mit den Gynäkologen im Austausch.

\section{Diskussion}

Seit 2017 steht der monoklonale AntiIL4/13-Rezeptor-Antikörper Dupilumab für die Therapie der mittelschweren bis schweren AD zur Verfügung [19]. In der Charité-Kohorte von insgesamt 104 Patienten wurde nach 3-monatiger DupilumabTherapie bei einem Drittel ein SCORAD (Scoring atopic dermatitis) 75 und bei zwei Dritteln ein SCORAD 50 erreicht [28]. Auch aus dem deutschen TREAT-Register werden therapeutische Erfolge gemeldet: durchschnittliche EASI-Reduktion nach 3 Monaten auf 74\% [1]. Gerade für neu eingeführte Arzneimittel ist die Beobachtung von Wirksamkeit und Sicherheit unter sog. Real-Life-Bedingungen von großer Bedeutung, um einen Eindruck zu gewinnen, ob sich die in randomisierten kontrollierten Studien erhobenen Daten replizieren lassen [28]. Real-Life-Daten können mit Beobachtungsstudien (NIS [nichtinterventionelle Studien]) sowie in Registern [1] erhoben werden. Es sei ausdrücklich darauf verwiesen, dass es sich in unserer Arbeit um eine beschreibende Fall- serie handelt, deren Limitation natürlich in der fehlenden Repräsentativität sowie im Fehlen einer internen Kontrollgruppe besteht. Die hier angewandte Methode ist die retrospektive Datenauswertung einer Kohorte (retrospektive Kohortenstudie; $[9,13])$. Diese Patientinnen und Patienten wurden unter keinem anderen Kriterium für die Therapie ausgewählt als dem vorgeschriebenen Krankheitsschweregrad (EASI, gelegentlich SCORAD, DLQI). Für die Patientenbetreuung, Dokumentation und Auswertung gab es selbstredend keinerlei finanzielle Zuwendungen durch die Pharmaindustrie. Ein Problem für die retrospektive Datenauswertung ergibt sich aus der unterschiedlichen Qualität der Dokumentation und damit bestehenden Datenlücken. Dies wird auch in unserer Patientenkohorte deutlich. Insgesamt fällt auf, dass die Schweregradnomenklaturen EASI und SCORAD (ebenso wie PASI [Psoriasis Area and Severity Index] für Psoriasis und UAS [Urtikaria-Aktivitäts-Score] für Urtikaria) eher selten in Schriftwechseln, wie z. B. Klinikbriefen, Verwendung finden. Sie könnten aber viel zu einem einheitlichen Verständnis über Schwere und Therapieverläufe beitragen. Wir haben für die Bewertung der Patienten hinsichtlich einer Dupilumab-Therapie immer die "Checkliste: Indikationsstellung zur Systemtherapie der Neurodermitis bei Erwachsenen“ [29] genutzt. Diese war und ist uns ein sehr wertvolles Instrument, weil sie die Vielfalt der Erkrankungsbilder durch viele "oder" bzw. "mindestens eines von ... Kriterien" abbildet. Nicht jeder EASI drückt den Schweregrad der Erkrankung authentisch aus, und eine Reihe unserer Patienten hat sich mit der Erkrankung abgefunden, was der relativ wenig beeinträchtigte DLQI zum Ausdruck bringt. Eine Herausforderung ergibt sich aus dem phasenhaften Verlauf der AD und der Tatsache, dass zum Termin der Vorstellung nicht immer der wirklich schwere Zustand zu dokumentieren ist. Und es stellt sich die Frage, ob man bei der Übernahme von Studienpatienten oder von Patienten aus Reha-Kliniken mit EASI $<10$ warten soll, bis der nächste schwere Schub stattfindet, um dann erst die Therapie zu starten. Wir haben uns in solchen Situationen entschlossen, einen „anamnestischen EASI“ anhand der Verläufe zu 
erheben und sorgfältig zu dokumentieren. Die Einrichtung der Spezialsprechstunde Immundermatologie für Patienten mit immunologisch bedingten chronisch entzündlichen Hauterkrankungen [16] hat dafür eine wichtige Struktur geschaffen. Leider zeigen entsprechende Auswertungen auch, dass die Betreuung solcher Patienten und insbesondere das Management der Biologikatherapien im Pauschalhonorarsystem für GKV(gesetzliche Krankenversicherung)-Patienten nicht annähernd kostendeckend abgebildet sind. Trotzdem waren es diese Sprechstunde und die damit verbundene Möglichkeit, den Patienten schnell und häufig Termine anbieten und so auf jede Situation reagieren zu können, die offensichtlich zu einer hohen Therapieadhärenz der Patienten beitrugen. In unserer Spezialsprechstunde werden inzwischen über 100 Patienten mit Systemtherapien (v. a. Biologika) behandelt. Der immer noch besorgniserregend schlechte Versorgungszustand der Patienten mit $A D$ ( $\bullet$ Tab. 1) sorgt dafür, dass sich nahezu wöchentlich ein neuer Therapiekandidat vorstellt. Abgesehen von den beachtlichen Arzneimittelkosten stellt sich eindeutig die Ressourcenfrage, denn die Sprechstunde in der derzeitigen Form (1 MFA, 1 Arzt für 1 Tag pro Woche) ist an ihre Grenzen gelangt und ein Ausbau betriebswirtschaftlich nicht vertretbar.

Die $A D$ als chronische Ekzemerkrankung mit Juckreiz ist oft assoziiert mit anderen, IgE-vermittelten Allergien wie Heuschnupfen, allergischem Asthma und Nahrungsmittelallergien [24-26]. Dies wird auch in unserer Kohorte deutlich. Insbesondere das allergische Asthma ist eine häufige Komorbidität (14/44 Patienten). Dupilumab ist neben der $A D$ auch für die Behandlung des Asthmas bzw. der Polyposis nasi (1/44 Patienten) zugelassen [30]. Unsere Patienten waren in pulmologischen Praxen diagnostiziert und mit entsprechenden Therapien versorgt worden. Wir informierten zeitnah über die Behandlung der Hautindikation mit Dupilumab. Eine Änderung der Asthmamedikation nahmen wir selbst nie vor (dies ist Sache der Pulmologen), sahen aber in allen Fällen eine Besserung, soweit vom Dermatologen beurteilbar. Ebenso pflegen wir eine gute Zusammenarbeit mit augenärztlichen Praxen, allerdings aus einem anderen Grund. Eine periorbitale Dermatitis mit und ohne Konjunktivitis und (seltener) Keratitis und Blepharitis sind als eine oft beobachtete Nebenwirkung der Therapie mit Dupilumab inklusive therapeutischer Hinweise beschrieben [3]. Derartige Symptome kamen in den Zulassungsstudien SOLO1 und SOLO2 sowie der CHRONOS und der CAFE-Studie bei 5-28\% der Patienten vor [21]. Das Auftreten oder die Exazerbation dieser Symptomatik ist allerdings nur für Patienten mit AD beschrieben und kommt bei Dupilumab-Behandlung des Asthmas oder der Polyposis nasi nicht gehäuft vor [27]. Aus früheren Arbeiten ist bekannt, dass $20-43 \%$ der Patienten mit AD an einer okulären Symptomatik leiden [3, 8]. Unsere Analyse diesbezüglicher anamnestischer Angaben, der Dokumentation in Klinikbriefen oder der Fotodokumentation durch die Patienten selbst zeigt, dass knapp die Hälfte (19/44) der Patienten unserer Kohorte von dieser Symptomatik betroffen war. Nur in dieser Gruppe fanden sich Patienten, bei denen es unter Dupilumab-Therapie zum Fortbestehen oder zu einer Verstärkung kam. Wir sahen nie ein Neuauftreten einer schweren Gesichtsbeteiligung mit Konjunktivitis unter Therapie, wo dies vorher nicht dokumentiert war. Wir bewerten dies daher eher als verzögertes Abheilen der atopischen Dermatitis im Gesicht und an/in den Augen. Möglicherweise handelt es sich hier um eine Sondergruppe von Patienten mit AD, begleitet von einer kontaktekzemartigen Hautbeteiligung im Gesicht durch Allergene, die von außen dorthin gelangen im Sinne einer "Airborne-Dermatitis" [15]. Die besondere Anfälligkeit von Patienten mit schwerer AD für Kontaktsensibilisierungen ist bekannt (Review: [23]). Der Großteil der Patienten mit diesem Erscheinungsbild hatte auch eine schwere $A D$ am Körper (EASI >15), bei 3 von 19 Patienten stand die schwere Gesichtsbeteiligung im Vordergrund, am übrigen Körper wurde ein EASI unter 10 gemessen. Insgesamt 6 unserer Patienten hatten unter Therapie eine ophthalmologische Konsultation, die zur Verordnung steroidhaltiger Augentropfen führte. Bei allen Patienten ergab sich unter Dupilumab im Verlauf der Therapie ab etwa Woche 18 bis 24 eine deutliche Besserung der Gesichts- haut sowie der okulären Symptomatik mit gelegentlicher Rezidivneigung. Es gab keinen Therapieabbruch. Sehr wohl gab es einen erhöhten Aufwand an Vorstellungsterminen, Überweisung, Kontakt zur anderen Facharztgruppe, Patientengespräch und -aufklärung. Wir werden möglicherweise bei Patienten mit einer solchen Vorgeschichte zukünftig andere Therapien anwenden.

Es gibt bei Atopikern IgE-vermittelte und "IgE-unabhängige" Entzündungsmechanismen. Die meisten Atopiker haben erhöhtes IgE mit "Polysensitization“ [7]. Bei 36 der 41 diesbezüglich untersuchten Patienten unserer Kohorte wurde erhöhtes IgE gemessen, daher würden sie zur Subgruppe der extrinsischen $A D$ zählen, bei denen die IgE-Spiegel mit dem Schweregrad der Erkrankung korrelieren sollen [22]. Dies können wir so nicht bestätigen, was aber auch an unserer begrenzten Fallzahl liegen mag. Im Unterschied zu anderen Autoren [2] konnten wir bei 4 von 5 unserer Patienten mit nicht erhöhten IgE-Spiegeln (intrinsische AD; [22]) durchaus spezifische Reaktivitäten messen. Diese Befunde hatten jedoch keine Beziehung zum Behandlungserfolg mit Dupilumab.

Dupilumab ist inzwischen eine etablierte Therapie für die AD. Sicherheit und Wirksamkeit wurden auch über einen mehrjährigen Verlauf dokumentiert [4]. Bei aller Sympathie für solche Daten stellt sich für uns zunehmend die Frage, wie groß die Kohorte der Dupilumab-behandelten Patienten noch werden wird. Da bisher kaum ein Patient die Behandlung beendet hat und sich fortlaufend neue Kandidaten dafür vorstellen, übersteigt die Kohortengröße demnächst die personellen Kapazitäten der Praxis [16]. Da wir aus immunologischer Sicht einen pathogenetischen Faktor blockieren und nicht seine Entstehung [18], müsste nach heutigem Verständnis lebenslang behandelt werden. Noch sind uns keine Ansätze bekannt, die es erlauben würden, eine Therapie ohne rasche Rezidive zu beenden. Vier unserer Patienten hatten aus logistischen Gründen (Dienstreise, Umzug) oder auf eigenen Wunsch eine Therapiepause. In jedem Fall sahen wir nach wenigen Monaten ein starkes Wiederaufflammen von Juckreiz und Entzündung. Die Wirksamkeit von Dupilumab in der Therapie der AD im Vergleich 
zu älteren Substanzen (MTX [Methotrexat], Cyclosporin A, Mycophenolat-Mofetil), wurde in einer Metaanalyse anhand von 39 randomisierten Studien mit einer Beobachtungsdauer von bis zu 16 Wochen untersucht [10]. Bei einem primären Endpunkt, derEASI-Reduktion, war Dupilumab mit einer Senkung gegenüber Placebo um durchschnittlich 11,3\% am erfolgreichsten - dies, wohlgemerkt, unter Studienbedingungen. In unserer Kohorte zeigt sich bei vergleichbarer Analyse ein etwas besserer Wert der EASI-Reduktion um 50, 75 oder $90 \%$ bei der Mehrheit der Patienten mit schnellem Ansprechen und im Therapieverlauf zumindest eines Jahres. Dies gilt auch für den Vergleich mit entsprechenden Daten aus den Zulassungsstudien (Phase 3) mit Kohortendaten aus der retrospektiven Analyse [21]. In der Phase-IIIStudie LIBERTY AD CHRONOS wurde nach 52 Wochen Therapie ein EASI 75 bei $65 \%$ der Patienten erreicht [6], in unserer Kohorte bei $86 \%$ (22 Patienten). Vergleiche einer beschreibenden Fallserie (unsere Kohorte) mit Ergebnissen großer Phase-IIIStudien sind nur sehr bedingt möglich, da es erhebliche Unterschiede z. B. in der Möglichkeit der Anwendung von Begleittherapien gibt. In unserer Kohorte ergab die diesbezügliche Auswertung in Woche 4 der Dupilumab-Therapie: 24/42 Patienten verwendeten regelmäßig oder sporadisch topische Glukokortikosteroide (TGK), davon 20 TGK Klasse II, 4 Klasse III. Topische Calcineurininhibitoren (TKI) wurden von 6/42 verwendet (einige in Kombination mit TGK). Insgesamt 14 von $42 \mathrm{~Pa}$ tienten nutzten „lediglich“ eine Basistherapie. Fünf Patienten nahmen sporadisch Antihistaminika ein. Eine ähnliche Untersuchung in Woche 52 zeigt, dass TGK, wenn überhaupt, nur noch sporadisch angewendet wurden. Möglicherweise hat auch die intensive Betreuung der Patienten durch MFA/Arzt in einer gut strukturierten Spezialsprechstunde die gute Wirksamkeit von Dupilumab unterstützt. Dies wäre ein weiterer Grund für eine bessere Honorierung eines guten Managements der Biologikatherapien in der dermatologischen Praxis. Neben dem Anti-IL4/13-Rezeptor-Antikörper Dupilumab werden weitere Zytokin- und Zytokinrezeptorantagonisten sowie eine ganze Gruppe von JAK-Inhibitoren entwickelt. Daher besteht die Hoff- nung, in den nächsten Jahren für die $\mathrm{Pa}$ tienten mit $A D$ ein ähnliches therapeutisches Repertoire anbieten zu können, wie es für die Psoriasis seit Jahren besteht [5, 28].

Zum Zeitpunkt der Entstehung dieses Manuskriptes kommt wohl keine klinische Arbeit ohne eine Stellungnahme zum Thema SARS-CoV-2-Pandemie aus. Wir haben bei allen Patienten die Dupilumab-Therapie fortgesetzt bzw. neu begonnen und sind damit entsprechenden Expertenempfehlungen [20] gefolgt. Dupilumab hat sein gutes Sicherheitsprofil in zahlreichen Studien bewiesen [11]. Zum Zeitpunkt Juni 2021 waren mehr als 50\% der Patienten unserer Dupilumab-Kohorte geimpft, zumeist mit dem RNA(Ribonukleinsäure)Impfstoff Comirnaty (BioNTech Manufacturing GmbH, Mainz, Deutschland). Wir haben keine Beeinträchtigung des Therapieverlaufes beobachtet.

\section{Fazit für die Praxis}

- Dupilumab ist eine sichere und effiziente immunmodulierende Therapie für die atopische Dermatitis (AD).

- Eine sorgfältige Dokumentation der Begründung der Therapieentscheidung sowie des Therapieverlaufes (EASI [Eczema Area and Severity Index], DLQI [Dermatology Life Quality Index], Pruritus-VAS [visuelle Analogskala] und Fotodokumentation zum Start und in Woche 4, 8, 16 und nachfolgend 1-mal pro Quartal) ist geboten.

- Bei Patienten mit schwerer Gesichts- und Augenbeteiligung gestaltet sich die Therapie aufwendiger. Daher können hier zukünftig andere Therapien zum Einsatz kommen.

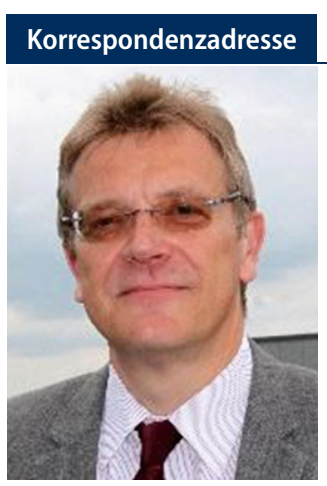

PD Dr. med. Sigbert Jahn

Dermatologische Praxis Dr. Herbst \& Kollegen Rheinstr. 7,64283 Darmstadt, Deutschland sigbertjahn@hotmail.com

Danksagung. Wir danken unseren Teammitgliedern, Frau Katharina Emmerich und Frau Tatjana Heller, für die großartige Unterstützung bei der Betreuung der Patienten und der Erhebung der klinischen Daten (EASI, DLQI, Fotodokumentation).

\section{Einhaltung ethischer Richtlinien}

Interessenkonflikt. S. Jahn hat Vortragshonorare, Reiseunterstützungen und Beraterhonorare u.a. von Sanofi, AbbVie, Almirall, Leo, Janssen erhalten. J. Föhr, E. Diamanti und M. Herbst geben an, dass kein Interessenkonflikt besteht.

Dieser Beitrag beinhaltet keine Studie an Menschen und Tieren. Es wurden retrospektiv klinische Daten erhoben, die im Praxisalltag zur Dokumentation erhoben wurden.

\section{Literatur}

1. Abraham S, Haufe E, Harder I et al (2020) The TREATgermany study group. Implementation of dupilumabin routine care of atopiceczema. Results from the German national registry TREATgermany. Br JDermatol 183:382-384

2. Akdis CA, Akdis M (2003) Immunologic differences between intrinsic and extrinsic types of atopic dermatitis. Clin Exp Allergy 33:1618-1621

3. Ascodi N, Thuraum S, Seegräber M et al (2019) Management of dupilumab-associated conjunctivitis in atopic dermatitis. J Dtsch Dermatol Ges 17:488-492

4. Beck LA, Thaci D, Deleuran et al (2020) Dupilumab provides favorable safety and sustained efficacy for up to 3 years in an open-label study of adults with moderate-severe atopic dernatitis. Am J Clin Dermatol 21:567-577

5. Bieber T (2019) Novel therapies based on the pathophysiology of atopic dermatitis. J Dtsch Dermatol Ges 17:1150-1161

6. Blauvelt $A$, deBruin-Weller $M$, Gooderham $M$ et al (2017) Long-term management of moderateto-severe atopic dermatitis with dupilumab and concomitant topical corticosteroids. (LIBERTY AD CHRONOS): a 1-Year randomized, double- 
blinded, placebo-controlled phase 3 trial. Lancet 389:2287-2303

7. Campana R, Dzore S, Mittermann I et al (2017) Molecular aspects of allergens in atopic dermatitis. Curr Opin Allergy Clin Immunol 17:269-277

8. Chen JJ, Applebaum DS, Sun GS et al (2014) Atopic keratokonjunctivitis: a review. J Am Acad Dermatol 70:569-575

9. Doll R (2001) Cohort studies. History of the method. II. Retrospective cohort studies. Sozial Präventivmed 46:152-160

10. Drucker AM, Ellis AG, Bohdanowicz M et al (2020) Systemic immunomodulatory treatments for patients with atopic dermatitis. JAMA Dermatol 156:659-667

11. Eichenfield LF, Bieber T, Beck LA (2019) Infections in dupilumab clinical trials in atopic dermatitis: a comprehensive pooled analysis. Am J Clin Dermatol 20:443-456

12. Gieler U, Gieler T, Milena E et al (2020) Skin and psychosomatics - psychodermatology today. JDtsch Dermatol Ges 18:1280-1298

13. Grimes DA, Schulz KF (2002) Cohort studies: Marching towards outcomes. Lancet 359:341-345

14. Grobe W, Bieber T, NovakN (2019) Pathophysiology of atopic dermatitis. J Dtsch Dermatol Ges 17:433-440

15. Handa S, De D, Mahajan R (2011) Airborne contact dermatitis-current persepctives in etiopathogenesis and management. Indian JDermatol 56:700-706

16. Jahn S, Föhr J, HerbstM (2020) Immunomodulators (Biologicals) in the treatment of patients with chronic dermatoses in the dermatological practice. Akt Dermatol 46:425-433

17. Jappe U, Beckert H, Bergmann K-C et al (2021) Biologics for atopic diseases: Indication, side effect management, and new developments. Allergolselect 5:1-25

18. Juntilla IS (2018) Tuning the cytokine responses: an update on Interleukin (IL)-4 and IL-13 receptor complexes. Front Immunol 9:888

19. Kraft M, Worm M (2017) Dupilumab in the treatment of moderate-to-severe atopic dermatitis. Expert Rev Clin Immunol 13:301-310

20. Schön MP, Berking C, Biedermann T et al (2020) COVID-19 und Immunregulation - von grundlegenden und translationalen zu klinischen Implikationen. J Dtsch Dermatol Ges 18:795-809

21. Simpson EL, Bieber T, Guttman-Yassky Eet al (2016) Two phase 3 trials of dupilumab versus placebo in atopic dermatitis. N Engl J Med 375:2335-2348

22. Suarez-Farinas M, Dhingra N, Gittler J et al (2013) Intrinsic atopic dermatitis (AD) shows similar Th2 and higher Th17 immune activation compared to extrinsic AD.J Allergy Clin Immunol 132:361-370

23. Uter W, Werfel T, White IR et al (2018) Contact allergy: a review of current problems from a clinical perspective. Int JEnviron Res Public Health 15:1108

24. Wollenberg A, Oranje A, Deleuran $\mathrm{M}$ et al (2016) ETFAD/EADV Eczema task force 2015 position paper on diagnosis and treatment of atopic dermatitis in adult and pediatric patients. J Eur Acad Dermatol Venerol 30:729-747

25. Weidinger S, Novak N (2016) Atopic dermatitis. Lancet 387:1109-1122

26. Werfel T, Allam JP, Biedermann T et al (2016) Cellular and molecular immunologic mechanisms in patients with atopic dermatitis. J Allergy Clin Immunol 138:336-349

27. Wollenberg A, Ariens L, Thurau S et al (2018) Conjunctivitis occuring in atopic dermatitis patients treated with dupilumab-clinical characteristics
Treatment of atopic dermatitis with dupilumab. A retrospective cohort analysis from dermatological practice

We present the results of a retrospective data analysis of a practice cohort of 44 patients with atopic dermatitis treated with the IL-4/13 receptor antibody dupilumab for up to 3 years. Patients were followed up over a period of 21 months during specialized consultation hours named Immunodermatology, which was established to guarantee comprehensive documentation. The patient's characteristics regarding age and sex distribution, severity and duration of disease were comparable with the patient collectives in large, pivotal studies. The therapeutic efficiency however was high (percentage of patients with EASI50, $-75,-90$ after 16 weeks: $94,84,60 \%$, respectively) and long lasting (86\% EASI90 after 52 weeks on therapy) under everyday conditions in the clinical setting. Approximately half of the patients had facial skin or eye involvement either in their history or at the start of treatment. This group of patients proved to need more and intense care because facial dermatitis and periocular dermatitis, which often involved conjunctivitis, took longer to heal, relapses occurred, and an additional topical treatment was often required. We did not observe any severe side effects during the 48 patient-years analyzed in this study. Dupilumab proved to be a safe and efficient treatment for atopic dermatitis in dermatological practice.

\section{Keywords}

Facial dermatitis · Immunodermatology · Interleukin-4/13 blockade · Immunomodulation . Periocular dermatitis

and treatment. J Allergy Clin Immunol Pract 6:1178-1780

28. Worm M, Francuzik W, Kraft M, Alexiou A (2020) Modern therapies in atopic dermatitis: biologics and small molecule drugs. J Dtsch Dermatol Ges 18:1085-1093

29. Augustin M, v. Werfel T, Kiedrowski R https:// www.teledermatologikum.de/wp-content/ uploads/AtopiTool-Checkliste-AM-TherapieNeurodermitis.pdf.Zugegriffen:27.Dez. 2020

30. Fachinformation Dupixent ${ }^{\circledR}$, Sanofi, 2021, Stand Januar

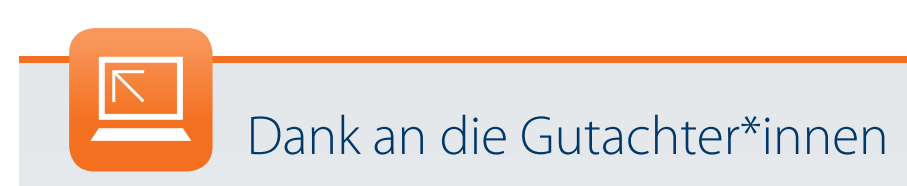

Für die Qualität und Objektivität der Beiträge sind neben den engagierten Autorinnen und Autoren auch die vielen qualifizierten Gutachterinnen und Gutachter maßgeblich, die im Rahmen des Peer-Review-Prozesses die Manuskripte inhaltlich-wissenschaftlich prüfen und Empfehlungen zur konkreten Verbesserung äußern. Allen Gutachterinnen und Gutachtern, die im vergangenen Jahr Manuskripte für diese Zeitschrift begutachtet haben, danken wir herzlich für die konstruktive und gewissenhafte Arbeit.

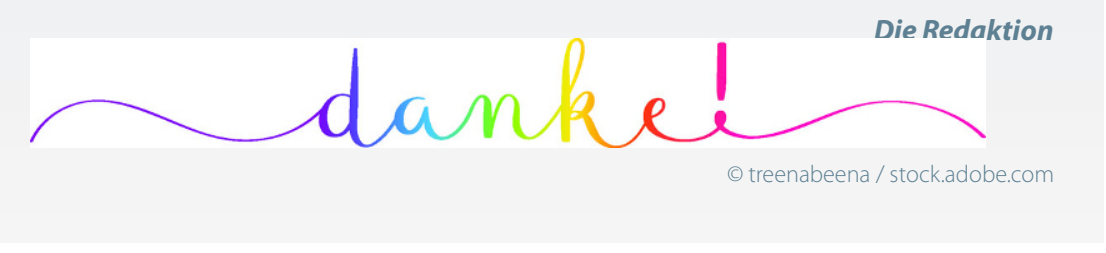


Hier steht eine Anzeige.

黑 Springer 